\section{OPEN}

SUBJECT AREAS:

MECHANICAL

ENGINEERING

APPLIED MATHEMATICS

Received

25 November 2013

Accepted

24 December 2013

Published

17 January 2014

Correspondence and requests for materials should be addressed to

V.L.P. (v.popov@tuberlin.de)

\title{
Analytic solution for the limiting shape of profiles due to fretting wear
}

\author{
Valentin L. Popov
}

Berlin University of Technology, 10623 Berlin, Germany.

We consider fretting wear due to tangential oscillations of two contacting bodies. For small oscillation amplitudes, the wear occurs only in a circular slip zone at the border of the contact area. With increasing number of cycles, the wear profile tends to a limiting form, in which no further wear occurs. Under assumption of a constant coefficient of friction, the limiting form of the wear profile does not depend on the particular wear criterion and can be calculated analytically. An explicit analytic solution is presented for arbitrary initial shape and illustrated for the cases of parabolic and conical shapes.

retting wear occurs if two bodies are pressed against each other and subsequently subjected to tangential oscillations with small amplitude. It is of importance for any technical system with joints subjected to oscillations. Very much attention was paid to fretting in such applications as fretting of tubes in steam generators and heat exchangers ${ }^{1-3}$, joints in orthopedics ${ }^{4}$, electrical connectors ${ }^{5}$, and dovetail blade roots of gas turbines $s^{6,7}$ as well as many others. Depending on particular properties of materials and on loading conditions, fretting wear can lead either to a progressive wear or to some final state in which no further wear occurs. The proof of the existence of the final state was done by Ciavarella and Hills ${ }^{8}$ for the case of an arbitrary two-dimensional (plane strain) contact problem. In the present paper, we show that their conclusions are also valid for threedimensional contacts of axis-symmetric bodies and derive the shape of the final, no-wear state.

Consider a rotationally symmetric profile which is brought into contact with a rigid surface and then oscillates in tangential direction with a given amplitude $u_{x}^{(0)}$. Under assumption of sticking condition, the tangential stress has a singularity at the border of the contact area ${ }^{9,10}$. In a contact with any finite coefficient of friction, this will lead to the appearance of a circular slip zone at the border of the contact area ${ }^{11-13}$ which leads to wear in this zone $^{8}$. In the present paper, we will assume that the friction can be described by a local formulation of the Amonton's law: The surfaces in contact are in the sticking state if tangential stress $\tau$ is smaller than normal pressure $p$ multiplied with a constant coefficient of friction $\mu$, and the tangential stress remains constant after the onset of sliding:

$$
\begin{aligned}
& \tau<\mu p, \text { stick } \\
& \tau=\mu p, \text { slip }
\end{aligned}
$$

At the circular border of the stick region with radius $c$, the critical condition $\tau(c)=\mu p(c)$ is fulfilled. Inside this region, the condition $\tau<\mu p$ is valid. Due to wear outside of the sticking region, the local pressure in the sticking region will increase and outside decrease further, independently of whether the experiment is done under conditions of constant normal force or constant indentation depth $d$. This will lead to a progressive wear outside of the region of stick. The wear process will advance until the pressure in the sliding region becomes zero. In this limiting state, the inner parts of the contact will still remain in the sticking state, while the wear rate in the outer parts of the contact tends to zero. The final state of no wear can be considered as a sort of "shakedown" state, in which no further inelastic processes occur. The detailed kinetics of the profile depends on the wear criterion used as well as on the loading conditions (controlled force or controlled indentation). In the most cases, the ReyeArchard-Khrushchov wear criterion is used, stating that the wear volume is proportional to the dissipated energy $^{14-16}$. According to this wear criterion, the wear rate vanishes if either the relative displacement $\Delta u_{x}$ of the bodies or tangential stress in contact is zero. In non-adhesive contacts, the latter means vanishing of the normal pressure $p$. The no-wear condition thus reads:

$$
\text { No wear condition : }\left\{\begin{array}{l}
\text { either } p=0 \\
\text { or } \Delta u_{x}=0
\end{array}\right. \text {. }
$$


From these conditions, it follows that the pressure in the final state is non-zero only inside the stick area and vanishes outside. The whole process and the condition (2) for the final state were formulated in detail by Ciavarella and Hills in Ref.. . They suggested that it should be possible, using this condition, to deduce the final shape. This is done in the present paper.

\section{Results}

Analytical solution for the final state with the method of dimensionality reduction (MDR). To find the final profile we use the method of dimensionality reduction $(\mathrm{MDR})^{17-19}$. This method allows solving the normal and tangential contact problems for axisymmetric bodies by mapping them to a one-dimensional contact of properly defined elastic foundation. We would like to stress, that in spite of analyzing a contact with one-dimensional elastic foundation, the MDR provides exact solutions for the initial three-dimensional contact problem. The corresponding proofs can be found in Ref. ${ }^{19,20}$ as well as in Chapters 17 and 18 of the monograph ${ }^{18}$. In the following, we shortly recapitulate the basics of the method of dimensionality reduction.

The main steps of the MDR are the following. Given a threedimensional profile $z=f(r)$, we first determine the equivalent onedimensional profile ${ }^{20}$

$$
g(x)=|x| \int_{0}^{|x|} \frac{f^{\prime}(r)}{\sqrt{x^{2}-r^{2}}} \mathrm{~d} r .
$$

The back transformation is given by the integral

$$
f(r)=\frac{2}{\pi} \int_{0}^{r} \frac{g(x)}{\sqrt{r^{2}-x^{2}}} \mathrm{~d} x .
$$

The profile (3) is pressed to a given indentation depth $d$ into an elastic foundation consisting of independent springs with spacing $\Delta x$ whose normal and tangential stiffness is given by

$$
\begin{aligned}
& k_{z}=E^{*} \Delta x \\
& k_{x}=G^{*} \Delta x,
\end{aligned}
$$

where $E^{*}$ is the effective elastic modulus

$$
\frac{1}{E^{*}}=\frac{1-v_{1}^{2}}{E_{1}}+\frac{1-v_{2}^{2}}{E_{2}},
$$

and $G^{*}$ the effective shear modulus

$$
\frac{1}{G^{*}}=\frac{\left(2-v_{1}\right)}{4 G_{1}}+\frac{\left(2-v_{2}\right)}{4 G_{2}} .
$$

$E_{1}$ and $E_{2}$ are the Young's moduli, $G_{1}$ and $G_{2}$ the shear moduli of contacting bodies, $v_{1}$ and $v_{2}$ are their Poisson-ratios. Note that throughout this paper, we assume that the contacting materials satisfy the condition of "elastic similarity"

$$
\frac{1-2 v_{1}}{G_{1}}=\frac{1-2 v_{2}}{G_{2}}
$$

guaranteeing the decoupling of the normal and tangential contact problems 9 . In particular, this condition is always satisfied in the important case of the contact between a rigid body and an incompressible elastomer (both sides of equation (8) are then zero).

The resulting vertical displacements of springs are given by

$$
u_{z}(x)=d-g(x)
$$

and the linear force density

$$
q(x)=E^{*} u_{z}(x)=E^{*}(d-g(x)) .
$$

The contact radius $a$ is given by the condition

$$
g(a)=d
$$

According to MDR rules, the distribution of normal pressure $p$ in the initial three-dimensional problem can be calculated using the following integral transformation ${ }^{18,20}$ :

$$
p(r)=-\frac{1}{\pi} \int_{r}^{\infty} \frac{q^{\prime}(x)}{\sqrt{x^{2}-r^{2}}} \mathrm{~d} x=\frac{E^{*}}{\pi} \int_{r}^{\infty} \frac{g^{\prime}(x)}{\sqrt{x^{2}-r^{2}}} \mathrm{~d} x .
$$

If the profile is moved tangentially by $u_{x}^{(0)}$, the springs will be stressed both in the normal and tangential direction, and the radius $c$ of the stick region will be given by the condition that the tangential force $k_{x} u_{x}^{(0)}$ is equal to the coefficient of friction $\mu$ multiplied with the normal force $k_{z} u_{z}(c)$ :

$$
G^{*} u_{x}^{(0)}=\mu E^{*}(d-g(c))
$$

Let us denote the initial three-dimensional profile as $f_{0}(r)$, the corresponding one-dimensional image as $g_{0}(x)$ and the limiting shakedown shapes as $f_{\infty}(r)$ and $g_{\infty}(x)$ correspondingly. As discussed above, the pressure outside the stick area must vanish in the limiting shakedown state: $p(r)=0$, for $r>c$. From (12), it follows that

$$
g^{\prime}(x)=0 \text { and } g(x)=\text { const }=g_{0}, \text { for } c<x<a .
$$

From the condition (11), it then follows that the one-dimensional profile in the shakedown state has the form

$$
g_{\infty}(x)=\left\{\begin{array}{l}
g_{0}(x), \text { for } 0<x<c \\
d, \text { for } c<x<a
\end{array} .\right.
$$

This shape is schematically shown in Figure 1. The three-dimensional limiting shape can now be calculated by the back transformation (4):

$f_{\infty}(r)=\left\{\begin{array}{l}f_{0}(r), \text { for } 0<r<c \\ \frac{2}{\pi} \int_{0}^{c} \frac{g_{0}(x)}{\sqrt{r^{2}-x^{2}}} \mathrm{~d} x+\frac{2}{\pi} d \int_{c}^{r} \frac{1}{\sqrt{r^{2}-x^{2}}} \mathrm{~d} x, \text { for } c<r<a\end{array}\right.$

Examples of limiting profiles are shown in Figure 2.

Limiting shapes for indenters with different initial shape. $I$. Parabolic indenter. The initial profile is in this case $f_{0}(r)=r^{2} /(2 R)$, and the corresponding one-dimensional MDR-image $g_{0}(x)=x^{2} / R$. The radius of the stick region is given by the condition (13):

$$
c=\sqrt{R\left(d-\frac{G^{*}}{E^{*}} \frac{u_{x}^{(0)}}{\mu}\right) .}
$$

According to (16), the limiting three-dimensional profile has the form

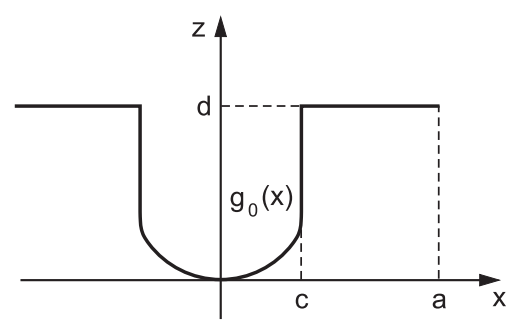

Figure 1 | One-dimensional MDR-image of the final "shakedown" profile. 

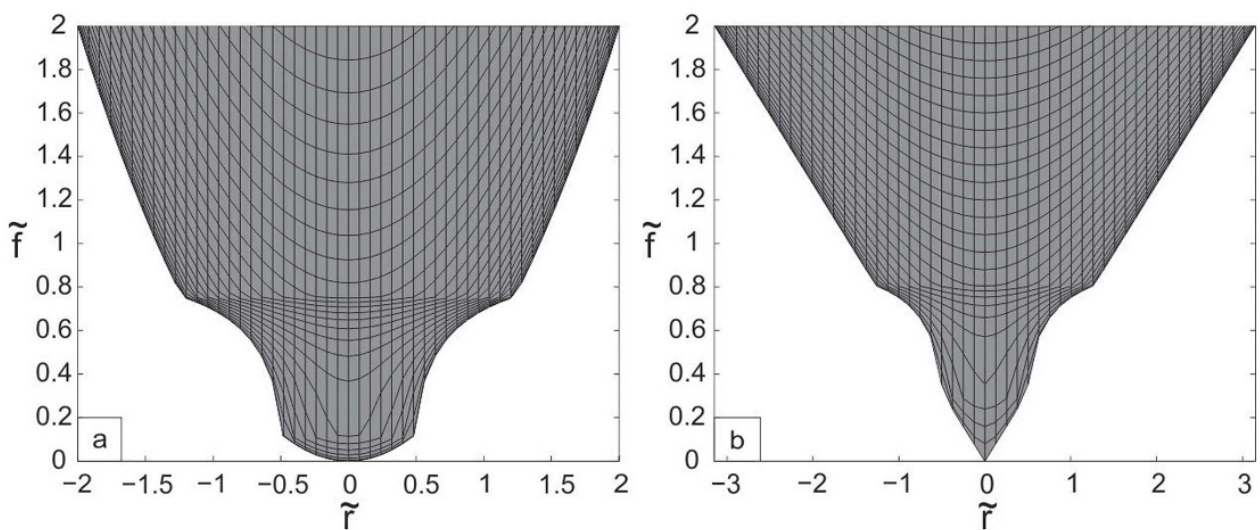

Figure $2 \mid$ Examples of limiting profiles with $\tilde{c}=0.5$ for initial (a) parabolic form and (b) conical form. Dimensionless variables are defined in Eq. (19).

$f_{\infty}(r)=\left\{\begin{array}{l}\frac{r^{2}}{2 R}, \text { for } 0<r<c \\ d-\frac{2}{\pi}\left(d-\frac{r^{2}}{2 R}\right) \arcsin \frac{c}{r}-\frac{r^{2}}{\pi R}\left(\frac{c}{r}\right) \sqrt{1-\left(\frac{c}{r}\right)^{2}}, \text { for } c<r<a\end{array}\right.$.

Normalizing all vertical coordinates by the indentation depth $d$ and horizontal coordinates by the contact radius of the initial profile, $a_{0}=\sqrt{R d}$,

$$
\begin{aligned}
& \tilde{f}=f / d, \tilde{d}=d / d=1, \\
& \tilde{r}=r / a_{0}, \tilde{x}=x / a_{0}, \tilde{c}=c / a_{0}, \tilde{a}=a / a_{0},
\end{aligned}
$$

we can rewrite these equations in the dimensionless form

$\tilde{f}_{\infty}(\tilde{r})=\left\{\begin{array}{l}\frac{\tilde{r}^{2}}{2}, \text { for } 0<\tilde{r}<\tilde{c} \\ 1-\frac{2}{\pi}\left(1-\frac{\tilde{r}^{2}}{2}\right) \arcsin \frac{\tilde{c}}{\tilde{r}}-\frac{\tilde{r} \tilde{c}}{\pi} \sqrt{1-\left(\frac{\tilde{c}}{\tilde{r}}\right)^{2}}, \text { for } \tilde{c}<\tilde{r}<\tilde{a}\end{array}\right.$

The non-dimensional form of the limiting profile thus depends only on one parameter $0<\tilde{c}<1$.

The contact radius, and thus the outer radius of the wear region, is given by the condition $\tilde{f}_{\infty}(\tilde{a})=\tilde{f}_{0}(\tilde{a})$ :

$$
1-\frac{2}{\pi}\left(1-\frac{\tilde{a}^{2}}{2}\right) \arcsin \frac{\tilde{c}}{\tilde{a}}-\frac{\tilde{a} \tilde{c}}{\pi} \sqrt{1-\left(\frac{\tilde{c}}{\tilde{a}}\right)^{2}}=\frac{\tilde{a}^{2}}{2} .
$$

In the limiting case $\tilde{c}=0$, the contact radius becomes $\tilde{a}=\sqrt{2}$.

The total force can be calculated as

$F_{N}=2 \int_{0}^{a} E^{*}(d-g(x)) \mathrm{d} x=2 \int_{0}^{c} E^{*}\left(d-x^{2} / R\right) \mathrm{d} x=2 E^{*}\left(d c-\frac{c^{3}}{3 R}\right)$

or, under consideration of (17),

$$
F_{N}=\frac{4}{3} E^{*} R^{1 / 2}\left(d-\frac{G^{*}}{E^{*}} \frac{u_{x}^{(0)}}{\mu}\right)^{1 / 2}\left(d+\frac{G^{*}}{2 E^{*}} \frac{u_{x}^{(0)}}{\mu}\right) .
$$

Profiles (20) are shown in Figure 3 for a representative set of parameters.

For $\tilde{c} / \tilde{r} \approx 1$, the exact solution (20) can be expanded in series:

$\tilde{f}_{\infty}(\tilde{r}) \approx\left\{\begin{array}{l}\frac{\tilde{r}^{2}}{2}, \text { for } 0<\tilde{r}<\tilde{c} \\ \frac{\tilde{r}^{2}}{2}+\left(1-\frac{1}{\pi}\left(2-\tilde{r}^{2}-\tilde{r} \tilde{c}\right)\right) \sqrt{2\left(1-\frac{\tilde{c}}{\tilde{r}}\right)} . \text { for } \tilde{c}<\tilde{r}<\tilde{a}\end{array}\right.$

While this equation is asymptotically exact for $\tilde{c} / \tilde{r} \rightarrow 1$, it gives a good approximation in the whole definition range of variables $\tilde{c}$ and $\tilde{r}$ with an accuracy not less than $15 \%$. Using this approximation, one can easily calculate the dependence of the outer wear radius $\tilde{a}$ on the inner wear radius $\tilde{c}$ :

$$
\tilde{a} \approx \sqrt{\left(\frac{\tilde{c}}{2}\right)^{2}+2}-\frac{\tilde{c}}{2}
$$

The normalized wear volume in the limiting state can be evaluated with the same accuracy as

$$
\tilde{V}_{\infty} \approx \pi(\tilde{c}-1)^{2}
$$

where $\tilde{V}_{\infty}=V_{\infty} /\left(d a_{0}^{2}\right)$, and $V_{\infty}$ is the wear volume.

II. Conical indenter. The initial profile is in this case $f_{0}(r)=r \tan \theta$, and the corresponding one-dimensional MDR-image $g_{0}(x)=$ $\frac{\pi}{2}|x| \tan \theta$. The radius of the stick region is given by the condition (13):

$$
c=\frac{2}{\pi \tan \theta}\left(d-\frac{G^{*}}{E^{*}} \frac{u_{x}^{(0)}}{\mu}\right) .
$$

The limiting three-dimensional profile has the form

$f_{\infty}(r)=\left\{\begin{array}{l}r \tan \theta, \text { for } 0<r<c \\ \left(r-\sqrt{r^{2}-c^{2}}\right) \tan \theta+d\left(1-\frac{2}{\pi} \arcsin \left(\frac{c}{r}\right)\right), \text { for } c<r<a\end{array}\right.$

Introducing the same dimensionless variables (19), but normalized by the initial contact radius $a_{0}=\frac{2}{\pi} \frac{d}{\tan \theta}$ for the cone, we come to the dimensionless presentation

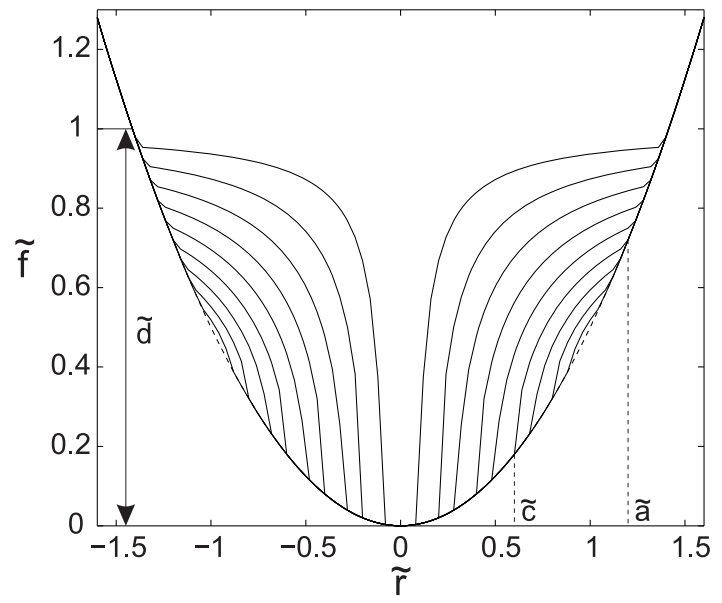

Figure $3 \mid 3 \mathrm{D}$ profiles in the final state according to equation (20). Parameters: 9 linearly increasing $\tilde{c}$ from 0.1 to 0.9 . 


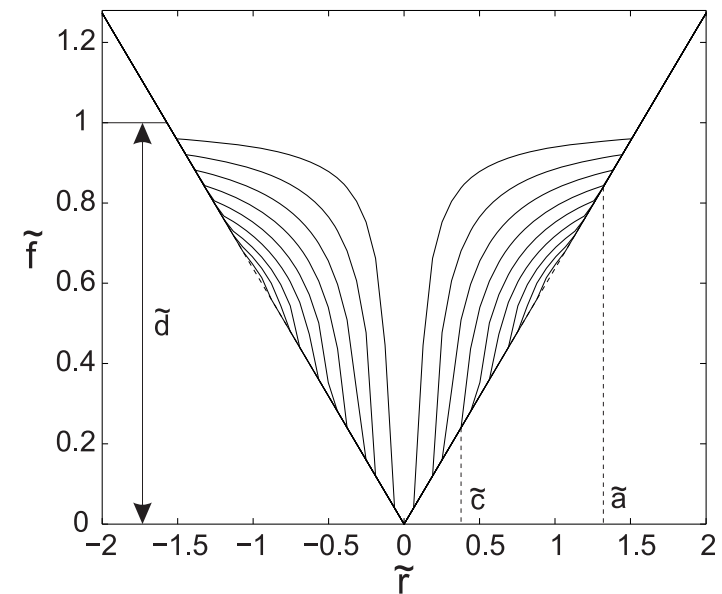

Figure $4 \mid 3 D$ profiles in the final state according to equation (29). Parameters: 9 linearly increasing $\tilde{c}$ from 0.1 to 0.9 .

$$
\tilde{f}_{\infty}(\tilde{r})=\left\{\begin{array}{l}
\frac{2}{\pi} \tilde{r}, \text { for } 0<\tilde{r}<\tilde{c} \\
\frac{2}{\pi}\left(\tilde{r}-\sqrt{\tilde{r}^{2}-\tilde{c}^{2}}\right)+\left(1-\frac{2}{\pi} \arcsin \left(\frac{\tilde{c}}{\tilde{r}}\right)\right), \text { for } \tilde{c}<\tilde{r}<\tilde{a}
\end{array}\right.
$$

The contact radius $a$ is given by the condition $f_{\infty}(a)=f_{0}(a)$, from which it follows that

$$
\frac{\pi}{2}-\arcsin \left(\frac{\tilde{c}}{\tilde{a}}\right)=\sqrt{\tilde{a}^{2}-\tilde{c}^{2}} .
$$

In the limiting case $\tilde{c}=0$, the contact radius becomes $\tilde{a}=\pi / 2$.

The total normal force is equal to

$$
\begin{aligned}
F_{N} & =2 \int_{0}^{a} E^{*}(d-g(x)) \mathrm{d} x=2 \int_{0}^{c} E^{*}\left(d-\frac{\pi}{2} x \tan \theta\right) \mathrm{d} x \\
& =2 E^{*}\left(d c-\frac{\pi}{4} c^{2} \tan \theta\right)
\end{aligned}
$$

or under consideration of (27),

$$
F_{N}=\frac{2 E^{*}}{\pi \tan \theta}\left(d^{2}-\left(\frac{G^{*}}{E^{*}} \frac{u_{x}^{(0)}}{\mu}\right)^{2}\right) .
$$

The profiles (28) are shown in Figure 4.

\section{Discussion}

In the present paper we considered wear of axis-symmetrical profiles due to tangential oscillations with small amplitude. Following former suggestion of Ciavarella and Hills, we have proven that there exists a final state in which the wear rate tends to zero. Using the method of dimensionality reduction, we have found an analytical solution for the shape in this final state in the closed integral form, Eq. (16). Explicit integration has been carried out for parabolic and conical profiles. The governing parameters of the final shape are indentation depth and radius of the stick region. We would like to discuss the main assumptions of this paper which affect the practical applicability of the theory: (a) using the Coulomb's law of friction with a constant coefficient of friction, and (b) assuming a local wear law, stating that the wear at each point does depend only on the pressure and relative tangential displacement at this point. Both assumptions are widely used but are known not to be completely correct: the Coulomb friction law is only approximately valid if the normal stress is not too small or too large ${ }^{10}$ and the derivations of the Khrushchev Archard wear relation show that the wear process is in reality always a non-local process. As the obtained solutions have sharp boundaries, at which the wear completely disappears and the normal stress becomes infinitely large, the above conditions are surely not applicable in the immediate vicinity of the boundary of the stick region. It is an interesting task for the future research to clarify the influence of these factors on the fretting wear and the very existence of the final state.

\section{Methods}

For analytical solution, we used the so-called Method of Dimensionality Reduction (MDR). The MDR is a simple technique of solving contact problems for axi-symmetrical bodies. In this method, a three-dimensional contact problem is replaced by a contact of a transformed profile with a properly defined elastic foundation. The most comprehensive description of the method with all necessary proofs is given in the recent monograph ${ }^{18}$ and in the dissertation ${ }^{19}$. The basics of the method are further described in $^{17,20}$. The MDR provides the exact threedimensional relations for indentation depth, contact radius and normal force as well as radius of the stick region and the tangential displacement for the tangential contact. Further, it allows restoring the correct three-dimensional distributions of normal and tangential stresses in the contact area, thus providing the complete solution of the three-dimensional problem.

1. Ko, P. L. Experimental Studies of Tube Frettings in Steam Generators and Heat Exchangers. J.?Press. Vessel Technol. 101, 125-133 (1979).

2. Fisher, N. J., Chow, A. B. \& Weckwerth, M. K. Experimental Fretting Wear Studies of Steam Generator Materials. J.?Press. Vessel Technol. 117, 312-320 (1995).

3. Lee, C. Y., Tian, L. S., Bae, J. W. \& Chai, Y. S. Application of influence function method on the fretting wear of tube-to-plate contact. Tribol. Int. 42, 951-957 (2009).

4. Collier, J. P. et al. Mechanicsms of Failure of modular prostheses. Clin. orthpaedics Relat. Res. 129-139 (1992).

5. Antler, M. Survey of contact fretting in electrical connectors. Components, Hybrids, Manuf. Technol. 8, 87-104 (1985).

6. Rajasekaran, R. \& Nowell, D. Fretting fatigue in dovetail blade roots: Experiment and analysis. Tribol. Int. 39, 1277-1285 (2006).

7. Ciavarella, M. \& Demelio, G. A review of analytical aspects of fretting fatigue, with extension to damage parameters, and application to dovetail joints. Int. J. Solids Struct. 38, 1791-1811 (2001).

8. Ciavarella, M. \& Hills, D. A. Brief Note: Some observations on the Oscillating Tangential Forces and Wear in General Plane Contacts. Eur. J. Mech. - A/Solids 18, 491-497 (1999).

9. Johnson, K. L. Contact Mechanics (Cambridge University Press, Cambridge, 1987).

10. Popov, V. L. Contact mechanics and friction. Physical principles and applications (Springer, Berlin, 2010).

11. Cattaneo, C. Sul contatto di due corpi elastici: distribuzione locale degli sforzi. Rend. dell'Accademia Naz. dei Lincei. 27, 342-348 (1938).

12. Mindlin, R. D. Compliance of elastic bodies in contact. J.?Appl. Mech. 16, 259-268 (1949).

13. Jäger, J. Axi-symmetric bodies of equal material in contact under torsion or shift. Arch. Appl. Mech. 65, 478-487 (1995).

14. Reye, T. Zur Theorie der Zapfenreibung. Der Civil. 4, 235-255 (1860).

15. Khrushchov, M. M. \& Babichev, M. A. Investigation of wear of metals. (Russian Academy of Sciences, Moscow, 1960).

16. Archard, J. F. \& Hirst, W. The Wear of Metals under Unlubricated Conditions. Proc. R. Soc. London A 236, 397-410 (1956).

17. Popov, V. L. Method of reduction of dimensionality in contact and friction mechanics: A linkage between micro and macro scales. Friction 1, 41-62 (2013).

18. Popov, V. L. \& He $\beta$, M. Methode der Dimensionsreduktion in Kontaktmechanik und Reibung. (Springer, Berlin, 2013).

19. He $\beta$, M. Über die Abbildung ausgewählter dreidimensionaler Kontakte auf Systeme mit niedrigerer räumlicher Dimension (Cuvillier-Verlag, Göttingen, 2011).

20. $\mathrm{He} \beta, \mathrm{M}$. On the reduction method of dimensionality: The exact mapping of axisymmetric contact problems with and without adhesion. Phys. Mesomech. 15, 264-269 (2012)

\section{Acknowledgments}

The author acknowledges many valuable discussions with $\mathrm{M}$. He $\beta$, M. Ciavarella, A. Filippov, A. Dimaki, A. Dmitriev, and Q. Li. This work was partially supported by Deutsche Forschungsgemeinschaft (DFG).

\section{Author contributions}

V.L.P. did all the work.

\section{Additional information}

Competing financial interests: The authors declare no competing financial interests. 
How to cite this article: Popov, V.L. Analytic solution for the limiting shape of profiles due to fretting wear. Sci. Rep. 4, 3749; DOI:10.1038/srep03749 (2014). cc)(1) (2) This work is licensed under a Creative Commons AttributionBY NC SA NonCommercial-ShareAlike 3.0 Unported license. To view a copy of this license, visit http://creativecommons.org/licenses/by-nc-sa/3.0 